\title{
New insights on the chemical modification of lignin:
}

\section{acetylation versus silylation}

\author{
Pietro Buono, ${ }^{1}$ Antoine Duval, ${ }^{2}$ Pierre Verge, ${ }^{1}$ Luc Averous ${ }^{2}$ and Youssef Habibi ${ }^{*}$ \\ ${ }^{I}$ Department of Materials Research and Technology (MRT), Luxembourg Institute of Science \\ and Technology (LIST), 5 avenue des Hauts-Fourneaux, L-4362 Esch-sur-Alzette, \\ Luxembourg. \\ ${ }^{2}$ BioTeam/ICPEES-ECPM, UMR CNRS 7515, Université de Strasbourg, 25 rue Becquerel, \\ Strasbourg Cedex 2 67087, France. \\ * To whom all correspondences should be addressed: Youssef.Habibi@list.lu
}

\section{Supporting Information}

5 pages, 3 tables and 6 figures 
Table S1. Quantification of the functional groups in the silylated lignins by ${ }^{31} \mathrm{P}\left(\mathrm{mmol} \mathrm{g}{ }^{-1}\right)$ and ${ }^{1} \mathrm{H}$ NMR $\left(\mathrm{mmol} \mathrm{g}^{-1}\right)$

\begin{tabular}{|c|c|c|c|c|c|c|c|c|c|}
\hline & \multirow[b]{2}{*}{$\begin{array}{c}\text { TBDMSCl } \\
\text { eq. }\end{array}$} & \multirow[b]{2}{*}{$\begin{array}{l}\text { Time } \\
\text { (h) }\end{array}$} & \multicolumn{6}{|c|}{ By ${ }^{31} \mathrm{P}$ NMR } & \multirow{2}{*}{$\begin{array}{c}\begin{array}{c}\text { By }{ }^{1} \mathbf{H} \\
\text { NMR }\end{array} \\
\text { TBDMS }\end{array}$} \\
\hline & & & Al-OH & $\begin{array}{l}\text { Cond. } \\
\text { Ph-OH }\end{array}$ & $\begin{array}{c}\text { G } \\
\text { Ph-OH }\end{array}$ & $\begin{array}{c}\text { H } \\
\text { Ph-OH }\end{array}$ & $\begin{array}{c}\text { Total } \\
\text { Ph-OH }\end{array}$ & $\mathrm{COOH}$ & \\
\hline SL & - & - & 1.80 & 1.61 & 1.09 & 0.46 & 3.16 & 0.90 & - \\
\hline Si-SL_01 & 0.4 & 18 & 1.07 & 1.28 & 0.85 & 0.35 & 2.48 & 0.55 & 0.67 \\
\hline Si-SL_02 & 0.8 & 18 & 1.05 & 0.88 & 0.53 & 0.21 & 1.62 & 0.46 & 1.78 \\
\hline Si-SL_03 & 1.2 & 18 & 0.44 & 0.15 & 0.07 & 0.03 & 0.25 & 0.23 & 2.71 \\
\hline Si-SL_04 & 2.5 & 18 & 0.30 & 0.00 & 0.00 & 0.00 & 0.00 & 0.32 & 2.38 \\
\hline Si-SL_05 & 5 & 18 & 0.29 & 0.00 & 0.00 & 0.00 & 0.00 & 0.45 & 3.37 \\
\hline Si-SL_06 & 1.2 & 1 & 0.69 & 0.52 & 0.30 & 0.15 & 0.98 & 0.35 & 2.85 \\
\hline Si-SL_07 & 1.2 & 3 & 0.58 & 0.27 & 0.15 & 0.05 & 0.47 & 0.28 & 3.20 \\
\hline Si-SL_08 & 1.2 & 5 & 0.65 & 0.33 & 0.16 & 0.08 & 0.57 & 0.36 & 3.41 \\
\hline Si-SL_09 & 2.5 & 1 & 0.44 & 0.21 & 0.00 & 0.00 & 0.21 & 0.41 & 3.40 \\
\hline Si-SL_10 & 2.5 & 3 & 0.39 & 0.00 & 0.00 & 0.00 & 0.00 & 0.33 & 3.48 \\
\hline Si-SL_11 & 2.5 & 5 & 0.63 & 0.00 & 0.00 & 0.00 & 0.00 & 0.46 & 2.77 \\
\hline
\end{tabular}

Table S2. Quantification of the functional groups in the acetylated lignins by ${ }^{31} \mathrm{P}\left(\mathrm{mmol} \mathrm{g}^{-1}\right)$ and ${ }^{1} \mathrm{H}$ NMR $\left(\mathrm{mmol} \mathrm{g}^{-1}\right)$

\begin{tabular}{|c|c|c|c|c|c|c|c|c|c|}
\hline & \multirow[b]{3}{*}{$\begin{array}{c}\mathrm{Ac}_{2} \mathbf{O} \\
\text { eq. }\end{array}$} & \multirow[b]{3}{*}{$\begin{array}{c}\text { Time } \\
\text { (h) }\end{array}$} & & & & & & & \multirow{3}{*}{$\begin{array}{l}\begin{array}{l}\text { By }{ }^{1} \mathbf{H} \\
\text { NMR }\end{array} \\
\text { Acetyl }\end{array}$} \\
\hline & & & \multicolumn{6}{|c|}{ By ${ }^{31}$ P NMR } & \\
\hline & & & Al-OH & $\begin{array}{l}\text { Cond. } \\
\text { Ph-OH }\end{array}$ & $\begin{array}{c}\text { G } \\
\text { Ph-OH }\end{array}$ & $\begin{array}{c}\text { H } \\
\text { Ph-OH }\end{array}$ & $\begin{array}{c}\text { Total } \\
\text { Ph-OH }\end{array}$ & $\mathrm{COOH}$ & \\
\hline SL & - & - & 1.80 & 1.61 & 1.09 & 0.46 & 3.16 & 0.90 & - \\
\hline Ac-SL_01 & 0.25 & 18 & 1.29 & 1.09 & 0.68 & 0.25 & 2.02 & 0.33 & 0.63 \\
\hline Ac-SL_02 & 0.5 & 18 & 1.19 & 0.82 & 0.50 & 0.17 & 1.50 & 0.34 & $n d$ \\
\hline Ac-SL_03 & 0.75 & 18 & 1.18 & 0.58 & 0.31 & 0.10 & 1.00 & 0.57 & 2.27 \\
\hline Ac-SL_04 & 1 & 18 & 1.11 & 0.43 & 0.17 & 0.05 & 0.65 & 0.40 & $n d$ \\
\hline Ac-SL_05 & 1.5 & 18 & 0.68 & 0.25 & 0.06 & 0.02 & 0.33 & 0.44 & 3.46 \\
\hline Ac-SL_06 & 2.5 & 18 & 0.00 & 0.00 & 0.00 & 0.00 & 0.00 & 0.90 & 4.31 \\
\hline Ac-SL_07 & 5 & 18 & 0.00 & 0.00 & 0.00 & 0.00 & 0.00 & 0.88 & 4.60 \\
\hline
\end{tabular}


Table S3. Solubility test for neat SL, acetylated SL (Ac-SL_06) and silylated SL (Si-SL_05).

\begin{tabular}{|c|c|c|c|c|c|}
\hline Solvent & $\begin{array}{l}\text { Boiling } \\
\text { Point }\left[{ }^{\circ} \mathrm{C}\right]\end{array}$ & $\begin{array}{c}\text { Polarity } \\
\left.\text { Index [ } P^{\prime}\right]\end{array}$ & SL & Ac-SL_06 & Si-SL_05 \\
\hline Water & 100 & 9.0 & - & - & - \\
\hline Water (NaOH 10\%) & $? ? ?$ & $? ? ?$ & +++ & - & - \\
\hline DMSO & 189 & 7.2 & +++ & ++ & $+/-$ \\
\hline DMF & 155 & 6.4 & +++ & +++ & +++ \\
\hline Pyridine & 115 & 5.3 & +++ & +++ & +++ \\
\hline Ethanol & 78 & 5.2 & $+/-$ & - & - \\
\hline Acetone & 56 & 5.1 & $+/-$ & +++ & +++ \\
\hline Methanol & 65 & 5.1 & $+/-$ & - & - \\
\hline 1,4 Dioxane & 101 & 4.8 & $+/-$ & ++ & ++ \\
\hline Ethyl Acetate & 77 & 4.4 & - & $+/-$ & +++ \\
\hline Chloroform & 61 & 4.1 & - & +++ & +++ \\
\hline THF & 65 & 4.0 & $+/-$ & +++ & +++ \\
\hline Dichloromethane & 41 & 3.1 & - & +++ & +++ \\
\hline Diethyl ether & 36 & 2.8 & - & - & +++ \\
\hline Hexane & 69 & 0.0 & - & - & - \\
\hline \multicolumn{6}{|c|}{+++ fully dissolved at $10 \%(\mathrm{w} / \mathrm{v})$} \\
\hline \multicolumn{6}{|c|}{++ fully dissolved at $2.5 \%(\mathrm{w} / \mathrm{v})$} \\
\hline \multicolumn{6}{|c|}{ +/- partially soluble } \\
\hline - insoluble & & & & & \\
\hline
\end{tabular}




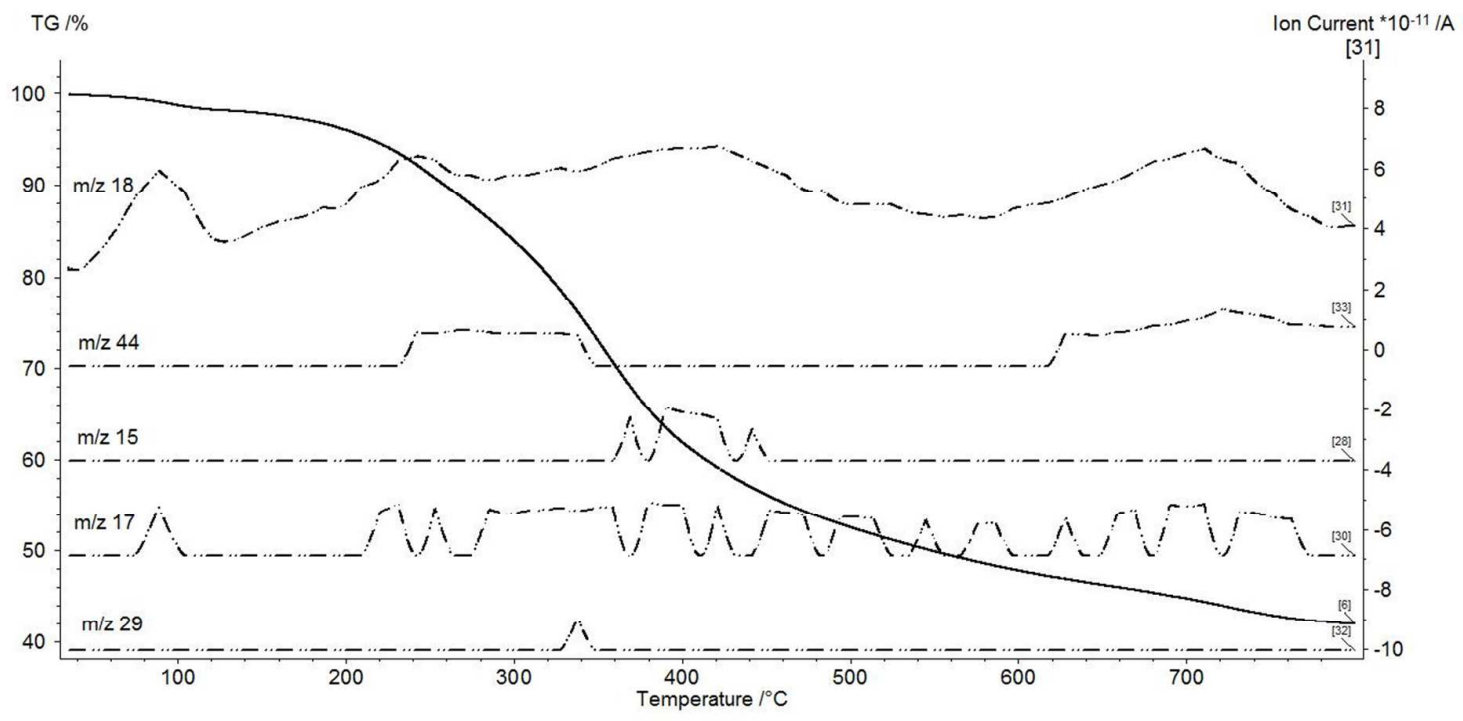

Figure S1. TGA coupled to MS of neat soda lignin (SL)

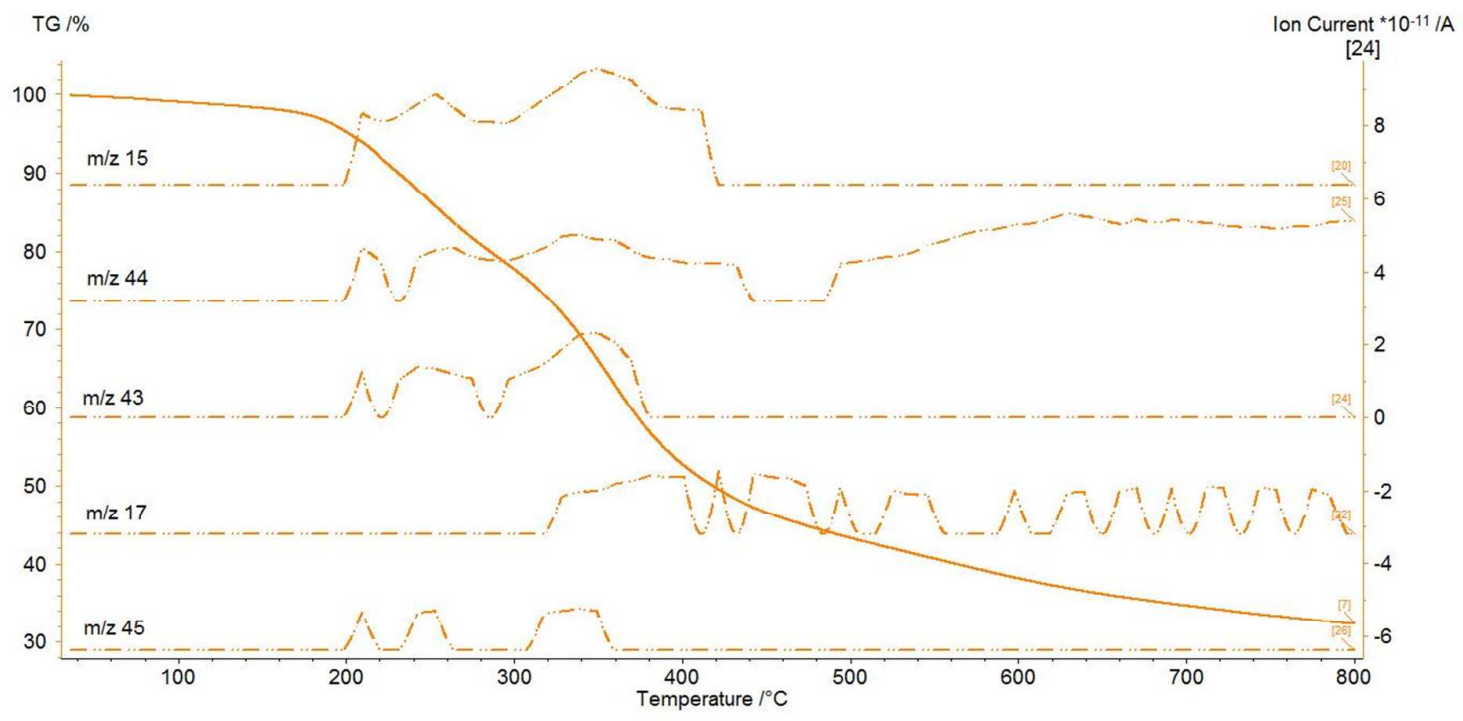

Figure S2. TGA coupled to MS of acetylated lignin (Ac-SL_06) 


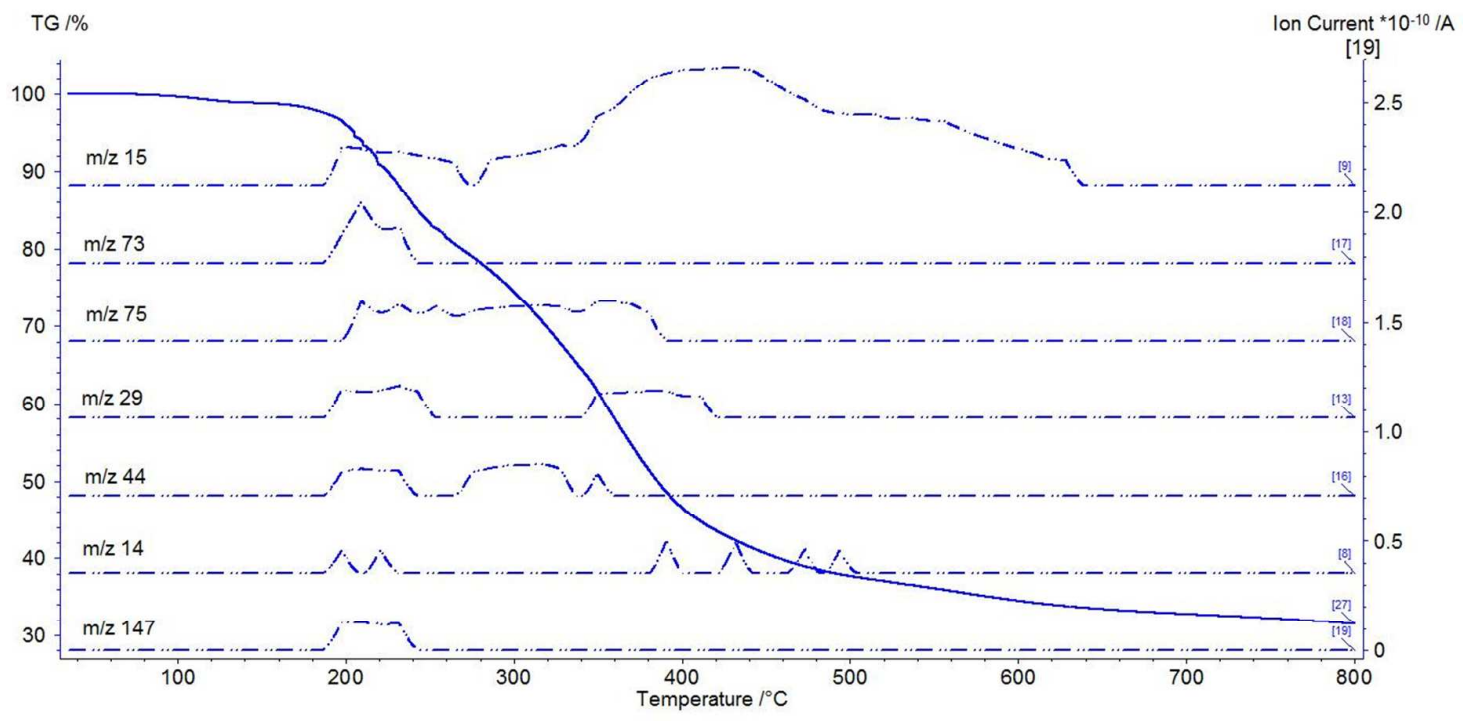

Figure S3. TGA coupled to MS of silylated lignin (Si-SL_05)

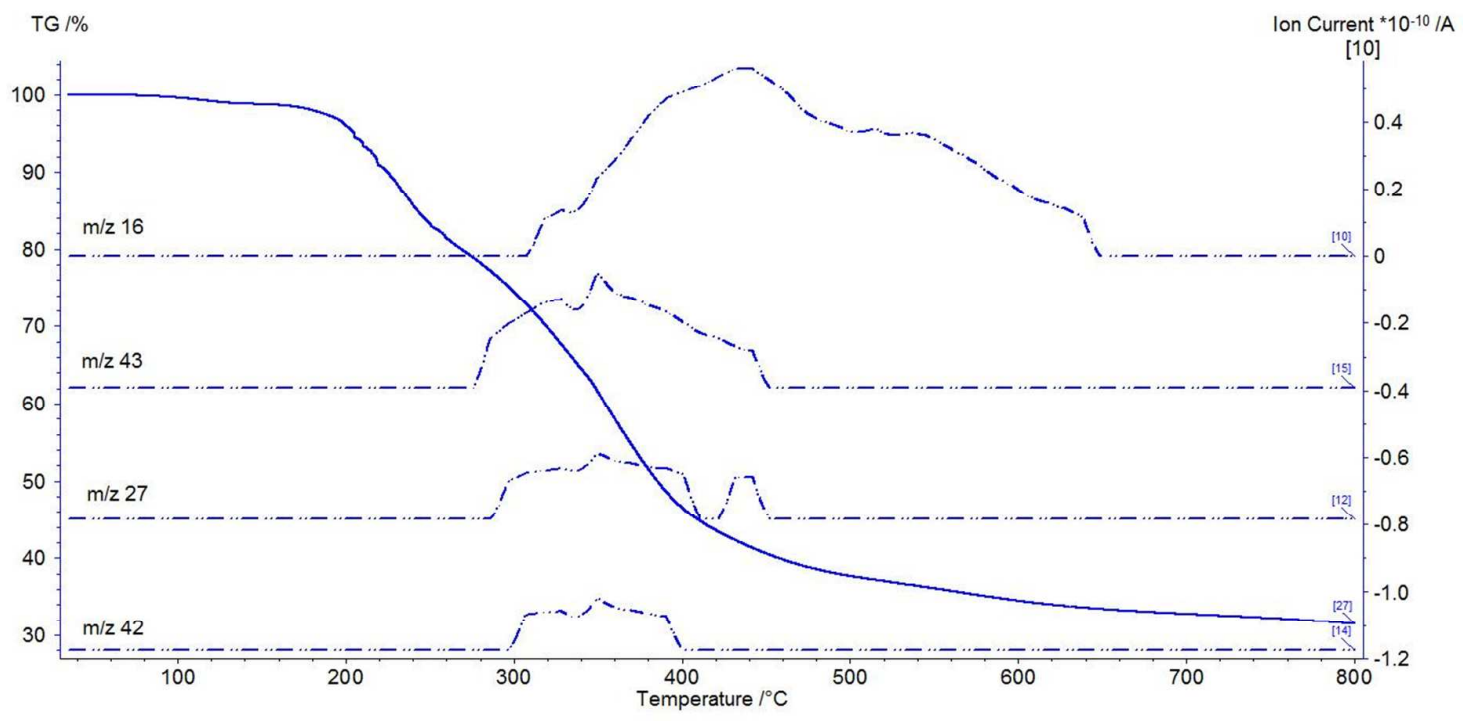

Figure S4. TGA coupled to MS of silylated lignin (Si-SL_05) (following) 


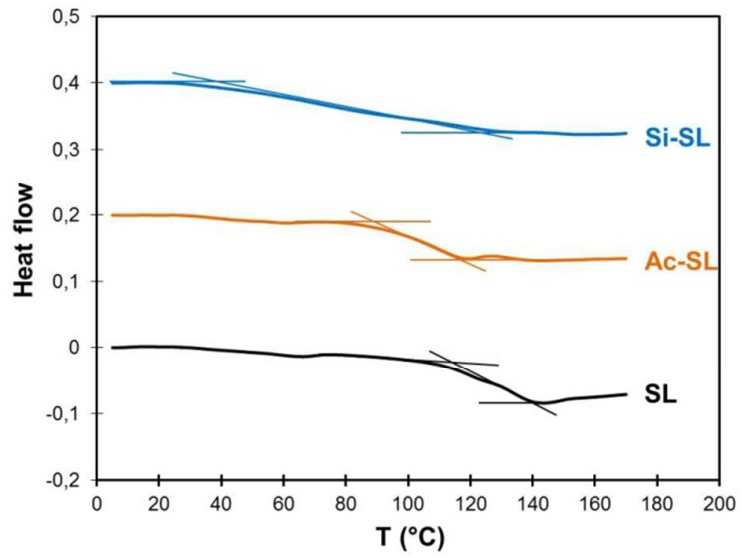

Figure S5. DSC traces of the neat (SL), acetylated (Ac-SL_06) and silylated lignins (Si-SL_05)
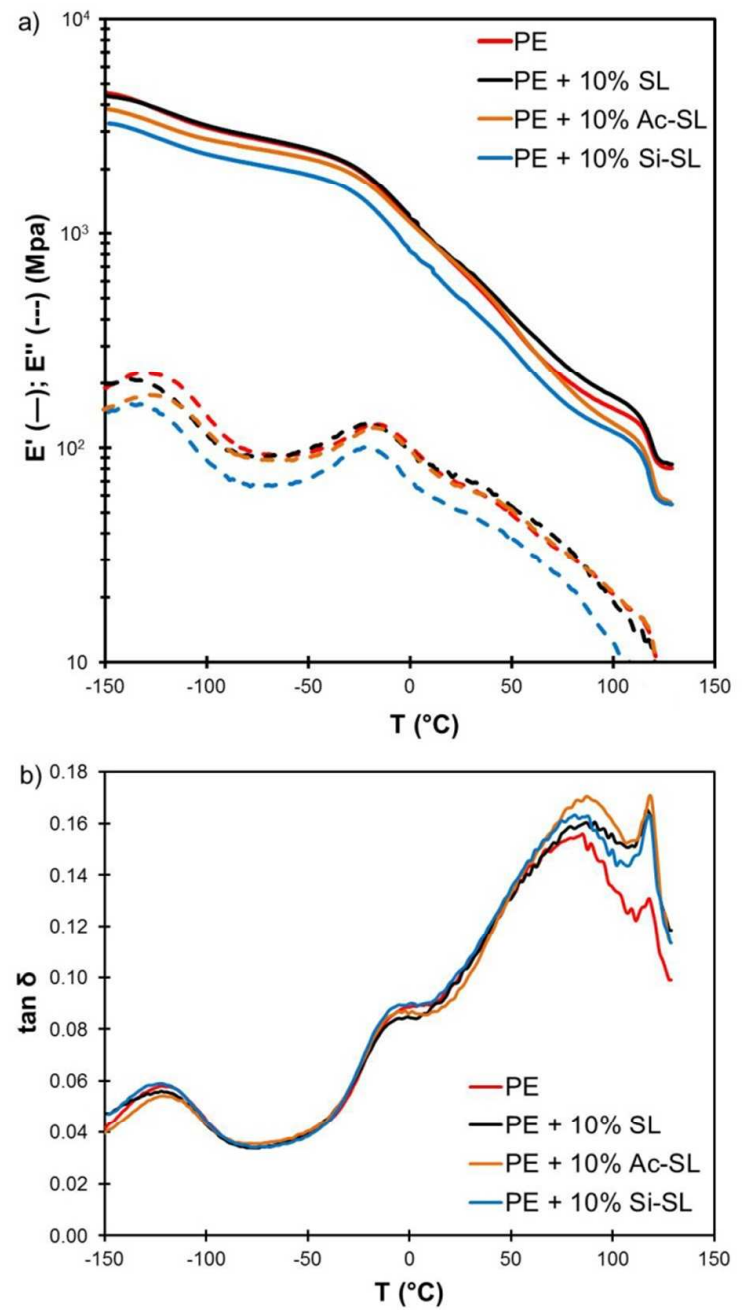

Figure S6. Dynamic mechanical analysis: storage $E^{\prime}$ and loss moduli $E^{\prime \prime}(a)$ and loss factor $\tan \delta(b)$ of LDPE and its blends with neat (SL), acetylated (Ac-SL_06) and silylated lignins (Si-SL_05). 